\title{
Women's accounts of the gestational diabetes experience - a South African perspective
}

\author{
L M Dickson, ${ }^{1} \mathrm{PhD} ; \mathrm{E}$ J Buchmann, ${ }^{2} \mathrm{PhD} ; \mathrm{S}$ A Norris, ${ }^{1} \mathrm{PhD}$
}

${ }^{1}$ MRC Developmental Pathways to Health Research Unit, Department of Paediatrics, University of the Witwatersrand, Johannesburg, South Africa

${ }^{2}$ Department of Obstetrics and Gynaecology, Faculty of Health Sciences, University of the Witwatersrand, Johannesburg, South Africa

Corresponding author: L M Dickson (lynnsaymd@diabeteswest.co.za)

Background. Considering the perspectives of African women affected by gestational diabetes mellitus (GDM) may facilitate the development of culturally sensitive interventions to address this public health concern.

Objective. To identify the personal experiences, challenges, coping strategies and health decisions made by urban indigenous South African women affected by GDM.

Methods. The experiences of urban African women $(n=10)$ previously affected by GDM were documented during focus group discussions. The Diabetes Conversation Map educational instrument was used to facilitate discussions. Sessions were audio-recorded, transcribed and thematically analysed.

Results. Participants experienced shock and fear on diagnosis of GDM, but family support and sharing their experiences with their peers provided relief from anxiety. They were aware of the inconsistent implementation of GDM diagnostic procedures at tertiary hospitals and community health clinics, which exacerbated their confusion. Despite their initial difficulty in adjusting to a diet perceived as unpalatable, adopting a healthier lifestyle was considered a positive consequence of a GDM diagnosis. Healthy lifestyle changes were partially retained post partum and were reported to have a positive effect on other family members. The participants had little awareness of their long-term risk of developing diabetes mellitus and the need for regular follow-up screening. Unexpectedly, the majority of participants concurred that being HIV-positive would be less stressful than living with diabetes.

Conclusion. Participants viewed being HIV-positive as preferable to having GDM, which may reflect the distress caused by inadequate pretest counselling, inconsistent health information, inconsistent screening practices and suboptimal support from healthcare professionals.

S Afr J Obstet Gynaecol 2020;26(1):22-28. https://doi/org/10.7196/SAJOG.2020.v26il.1534

The World Health Organization (WHO) estimates that, globally, $16 \%$ of pregnancies are affected by gestational diabetes mellitus $(\mathrm{GDM}){ }^{[1]}$ Women diagnosed with GDM are at high risk of poor perinatal outcomes, and both mother and child are at high risk of developing type 2 diabetes in later years. ${ }^{[2,3]}$ Lifestyle interventions are effective treatment for GDM in as many as $90 \%$ of those affected. ${ }^{[4]}$ These complex interventions typically include dietary advice, exercise, diabetes education and self-monitoring of blood glucose. Diabetes education and sustained healthy lifestyle choices are also important components of long-term diabetes prevention programmes. ${ }^{[5-8]}$ However, intervention strategies proven effective in developed countries are not necessarily directly transferable to low-resource settings. ${ }^{[9]}$ Culturally appropriate dietary advice and diabetes education are associated with sustained improved blood glucose control. ${ }^{[10-12]}$ Locally available skills and resources and pre-existing circumstances and attitudes may also affect GDM interventions and implementation strategies. ${ }^{[13,14]}$ Locally acceptable interventions that can be applied in low-resource settings are urgently needed to support women affected by GDM. ${ }^{[1]}$

Consideration of the experiences and expectations of local women may strengthen the development or adaptation of existing GDM interventions and help appropriately frame implementation strategies. ${ }^{[14-17]}$ It is known that cultural differences exist in women's experiences of GDM. ${ }^{[18]}$ Shock, anxiety, uncertainty, feelings of abandonment and a need for support in adapting to GDM have been reported. ${ }^{[18-20]}$ African women living in developed countries are reported to experience increased pregnancy-associated anxiety, with greater difficulty adjusting to GDM treatment. ${ }^{[21,22]}$ To our knowledge, this is the first qualitative research report of the GDM experiences of indigenous African women in an urban African setting. The purpose of our study was to identify the personal experiences, challenges, coping strategies and health decisions made by urban indigenous women affected by GDM in South Africa (SA).

\section{Methods \\ Setting and study participants}

This study was conducted at the Chris Hani Baragwanath Academic Hospital (CHBAH) in Soweto, Johannesburg, between December 2015 and February 2016. Participants were recruited from the Soweto First 1000 Days Cohort (S1000 cohort). This cohort of women are followed-up intensively during pregnancy, at birth and after pregnancy. All were screened for GDM at 26 - 29 weeks' gestation using a $75 \mathrm{~g}$ oral glucose tolerance test. Women with preexisting type 1 or type 2 diabetes mellitus and those below the age of 18 years were excluded from the cohort. Once diagnosed with GDM, women were referred to the CHBAH Diabetes in Pregnancy clinic for treatment.

A purposive sample of $\mathrm{S} 1000$ participants known to have had GDM in the previous 24 months was selected through telephonic recruitment $(n=15)$. Approval to conduct the study was granted 
by the University of the Witwatersrand Human Research Ethics Committee (ref. no. M150365). Participants gave written informed consent.

\section{Study design and protocol}

This study forms part of a larger study concerned with the development of an SA-applicable universal GDM screening programme and GDM lifestyle intervention suitable for use in a low-resource setting. ${ }^{[23,24]}$ The 'Healthy eating and keeping active' Diabetes Conversation Map (DCM), which is an International Diabetes Federation-approved educational instrument, was used both as a conceptual framework and to facilitate semistructured focus group discussions (FGDs). The DCM is a verbal, visual and highly interactive programme designed for delivery to groups of patients. It promotes the understanding of diabetes and how to manage the disease, explores the emotional aspects of diabetes and promotes self-management of diabetes over a lifetime. It has been shown to be as effective as other programmes with a more didactic educational style. ${ }^{[25]}$ The first author, who was trained in the use of the DCM instrument by a certified facilitator, served as the FGD moderator. Each participant attended a single FGD and the purpose of the FGD, as well as the role of the moderator, was explained at the outset. Discussions were conducted in English, but women were encouraged to speak in their own language. Two research assistants served as interpreters when language was a barrier to participation. Each FGD lasted 60 - 75 minutes. A structured script was developed to establish consistency in the data collection process.

\section{Data collection and analysis}

Clinical data were collected from participants' records, including age, parity, body mass index, family history of diabetes and their past obstetric history. Transcriptions of the audio-recorded FGDs were analysed thematically for commonalities and differences between participants' responses. Qualitative software was not used in this analysis. Themes and subthemes were derived from the data and unexpected themes were identified and noted. The context, frequency and intensity of the participants' comments guided the selection of quotations that were considered to reflect the group experience. This thematic analysis was validated by a second researcher.

After preliminary analysis of the data, participants were invited to a one-off formal checking session. Results were presented and the participants were invited to make further comments. Their level of agreement with the preliminary findings was noted, but no additional themes were identified.

\section{Results \\ Participants}

Of the 15 women who were invited, 10 agreed to participate. Three FGDs were scheduled to accommodate the participants and the number of participants per discussion varied between two and six. Eight of the ten participants attended the checking session, held several weeks later. All participants were urban black African women, in keeping with this hospital's patient profile. Their mean age was 35 years, with a mean body mass index of $26.8 \mathrm{~kg} / \mathrm{m}^{2}$ and half of them had a positive family history of diabetes mellitus. Adverse previous pregnancy outcomes were common: five participants had at least one previous miscarriage and one had a stillbirth before her most recent pregnancy.

\section{Qualitative data presented as themes and subthemes}

Theme 1: Participants' responses to their diagnosis

Participants were aware of inconsistencies in applying diagnostic procedures and criteria for GDM at the research unit, the CHBAH antenatal clinic and the community health clinic. Some reported disagreement between doctors regarding which diagnostic criteria to apply, which left them unconvinced that they had GDM. Participants expressed feelings of shock and fear because their diagnosis was unexpected. Previous miscarriage or stillbirth was associated with the possibility of undiagnosed GDM. Participants who had experienced previous pregnancy losses were reminded of these by their diagnosis and felt responsible because of 'not eating right'. This exacerbated participants' anxiety, which often persisted throughout their pregnancy.

At the checking session, all participants agreed that there should be more awareness about GDM in the community, with increased availability of screening. However, some women were ambivalent and indicated that they would have preferred not to know their diagnosis.

\section{Subtheme: Confusion, disbelief}

Group 2:

'The research unit said I am diabetic, but doctors at Bara [CHBAH] also said I am not diabetic, but the professor said I am diabetic. So, I don't know which was, but they gave me some tablets for diabetes.'

'This doctor said, "No, this is for the fourth time now you have got positive results for glucose in your urine test; you must do this OGTT [oral glucose tolerance test] test." Already I had tested at the research unit and it was positive. When I was supposed to start my diabetic treatment that's when I went to theatre for an emergency caesar because the baby was in distress.'

\section{Subtheme: Shock, fear, anxiety}

Group 1:

'The worse fear was losing the baby because I didn't know what made me lose the first baby. They told me that diabetes could make me lose this baby'

Group 2:

'It was stressful because father has diabetes and [I] didn't want to inject myself like my father and have diabetes my whole life.'

'If I was attending a local clinic maybe I could have died. I might have lost the baby because I wouldn't have known I had diabetes.'

Group 3:

'My mother has diabetes, so I knew what to expect.'

\section{Subtheme: Self-blame, guilt}

Group 2:

'I maybe made mistakes with the baby I lost. Maybe I didn't eat right. Maybe I didn't try my best. Maybe I got gestational diabetes that time. I don't know.

Theme 2: Participants' experiences of the healthcare service Most participants were admitted to the obstetric ward for initiation of treatment upon diagnosis. Participants were vocal and emotive 
in expressing dislike of their inpatient experience. Suboptimal communication between the research unit and the CHBAH staff about the purpose of the GDM research project caused frustration. Patients bore the brunt of the nurses' frustration, who complained that the women were being admitted for treatment despite the shortage of hospital beds.

Although participants were aware of resource shortages and the inequity in distribution of healthcare services between the community clinic and the hospital, they would have preferred attending antenatal services at the community clinic because it was less crowded and closer to their homes (incurring lower transportation costs). At the checking session, all participants agreed that the quality of regular check-ups at community clinics should improve. Most were of the opinion that the diagnosis of GDM was likely to be missed at a community clinic. The inconsistency of health information to support them during their pregnancies also left them confused and insecure.

\section{Subtheme: Experience of hospital admission}

Group 2:

'When we were admitted, our motivation was that we didn't want to stay longer so we drank a lot of water and ate their food and then we would be discharged.'

\section{Subtheme: Interactions with healthcare professionals Group 1:}

'The nurses shouted at me because they believed that I am not diabetic, and they were also complaining about this study ...'

\section{Subtheme: Awareness of resource shortages}

\section{Group 2:}

'It is full, it is full, the patients are sitting on benches the whole day, taking medication from the bench, even those who have [high] blood pressure. But if you are diabetic, they make amends for you to get a bed quickly.'

Group 3:

'They sent me home to test myself, because the hospital was full.'

\section{Subtheme: Inconsistent health information}

Group 1:

'I did not receive education while admitted to hospital and did not receive special treatment.'

\section{Group 2:}

'I was told that my baby would be big, but my baby was small, and the nurses shouted at me in the labour ward, asking me why was I taking medicine for diabetes because now my baby was too small and premature.'

\section{Theme 3: Lifestyle adjustments during pregnancy}

Adjusting to the prescribed diet was the most challenging aspect of treatment. Some participants felt that the diet was unpalatable. Some tolerated a temporary loss of dietary autonomy because changes were presumed necessary only for the duration of their pregnancy. Those who were given the opportunity to consult a dietitian felt better able to cope. Overall, changing to a healthy eating plan was considered a positive consequence of their GDM diagnosis. They learnt that exercise helps to control blood sugar levels and they incorporated exercise into their daily activities.
Participants who had a family member living with diabetes felt better able to cope. The wellbeing of their baby strongly motivated all participants to adopt and maintain healthy choices during their pregnancies.

At the checking session, participants agreed that eating unhealthy food 'makes you tired'. Although small meals and including fruit and vegetables in the diet would be ideal during pregnancy, these choices were generally considered to be more expensive.

\section{Subtheme: Unpalatable diet}

Group 1:

'The food you have to eat is like eating cardboard, a piece of paper that is only placed in water. There is no taste.'

Subtheme: Loss of autonomy

Group 1:

'It was a hardship for me having to eat that food because I was forced.'

Group 3:

'I had to adjust because there was nothing I could do.'

\section{Subtheme: Dietary adjustment difficulties}

Group 1:

'I attended sessions with the dietitian and went home relieved and empowered that, you know what, I can actually manage this.'

Group 2:

'I am eating spinach and trying by all means. I have a little garden now where I planted a bit of greens.'

'First I was so stubborn, I didn’t want to change.'

Subtheme: What is healthy eating?

Group 1:

‘.. lots of vegetables, low-fat milk, low-fat cooking techniques like boiling and roasting, reducing spices, no sauces, diluting fruit juice.'

Group 2:

'The diabetic diet is not much different from my usual diet in that I eat vegetables and fruits although I never stopped eating fatty foods.'

Group 3:

'When I am finished eating unhealthy food, I feel tired.'

\section{Subtheme: Healthy eating is expensive}

Group 3:

'Diabetes food is more expensive as you can no longer eat pap [maize meal], which makes diabetes complicated, especially with food prices going up, and if you don't eat in a proper manner you become sick.

\section{Subtheme: Benefits of exercise}

Group 1:

'I would recommend exercise to another pregnant woman with gestational diabetes although I did not exercise myself.'

\section{Group 2:}

'When I go to work, I walk to the station. Sometimes when I am late, I have to run because the train is there and if you are not at the station it will leave. So, for me I don't have to go to gym. 


\section{Theme 4: Women's need for support during pregnancy}

Most participants said that they had additional family support after being diagnosed, which made it easier to cope with the challenges of treatment; a minority said they had to cope with everything on their own. Participants acknowledged they had benefitted from informal peer support, both when they were admitted to hospital and during their clinic visits. In the waiting area of the clinic they became acquainted with one another and started sharing 'tips'. Sharing experiences with others who were 'in the same boat' helped to ease their anxiety. A common sentiment was that 'talking heals'.

At the checking session, participants unanimously agreed that although support from family, friends and peers was important, the individual was ultimately responsible for her own health and the changes she had made.

\section{Subtheme: Support from partner or family}

Group 1:

'My mother-in-law was diabetic, so she helped me to eat correctly, which made it easier.'

\section{Group 2:}

'When your partner or family does not want the same as you it makes following the diet more difficult, especially when they eat takeaways in front of you.'

\section{Group 3:}

'My brother, who has diabetes, said it is not a life sentence. Then he told me that he will help me, and I was okay'.

\section{Subtheme: Support from peers}

Group 1:

'I believe that talking heals. Sitting with a group of women who are going through the same experiences eases your anxiety levels. Knowing that you are not alone helps.'

\section{Group 2:}

'Sharing experiences and things that work for you with others and hearing their ideas and experiences helps.'

\section{Subtheme: Self-reliance}

Group 2:

'I think it has to come out from you. It is a decision that you have to make to take care of yourself.'

\section{Theme 5: Participants' understanding of diabetes}

Participants who had a family member with diabetes had a better understanding of the disease, the different types of diabetes and the underlying causes of the disease. A few participants were fatalistic regarding their future risk of developing diabetes mellitus. Although they were aware of the importance of healthy eating, they did not maintain the changes postpartum, because the diet was not aligned with their personal preferences. Not all participants had undergone an oral glucose tolerance test at 6 weeks postpartum, although this is clinically indicated. One of the participants was tested, but 6 months later still had not been informed of the results. Those who were aware of their future risk of developing diabetes mellitus were unsure where to go for continued care.

\section{Subtheme: Diabetes knowledge}

Group 1:

'I think that is the reason why you have it is because your family members have it. Not everybody from the family will get it. Like from my family it is only me and we are four, right. It chose me.'

Group 2:

'I was pregnant and I was telling people I am diabetic, they said, "Maybe they made a mistake. How can you be diabetic when you are so thin?" Because they think diabetes is only for big people.'

\section{Subtheme: Maintaining healthy choices}

Group 1:

'So, I was eating healthy and I said I think I am doing well, so why change and go back? It is like I have been eating like this forever.'

'My husband liked eating pap [maize meal] and meat; I was only doing vegetables for myself. But I managed to change him as well.'

Group 2:

'After the pregnancy I am back to my usual diet but [with] less sugar intake.'

\section{Group 3:}

'I will start eating the diet again once I have diabetes. I don't want to lie. I can't live with those kinds of foods every day of my life forever.'

\section{Subtheme: Awareness of diabetes risk \\ Group 1:}

'I thought about my mother ... I used to see her taking these pills every day and stuff and I said I don't want to go to that stage.'

\section{Group 3:}

'... having family members who have passed away from diabetes makes me realise I want to be around for the people I love.'

\section{Theme 6: An unexpected theme}

In two of the three FGDs, participants said that it would be less stressful to be infected with HIV than to have diabetes. At the checking session, all but one of the participants agreed that living with HIV would be less stressful than living with diabetes.

\section{Subtheme: Being HIV-positive is preferable to having diabetes Group 1: \\ 'I would rather be HIV-positive than being diabetic. The stress ...'}

Group 2:

'Having HIV is better than having diabetes, because diabetes is stressful.'

\section{Discussion}

To our knowledge, this is the first qualitative study of indigenous African women's experience of GDM conducted in a low- and middle-income setting. There are both commonalities with and differences from the experiences reported in other, international studies. 


\section{Healthcare professionals' interaction with participants}

Patients' confusion and disbelief regarding their GDM status were exacerbated by witnessing the professional discourse on the local implications of the change in GDM diagnostic criteria during clinical ward rounds. They were also aware of inconsistent screening practices, namely using either urine testing or the oral glucose tolerance test. Our participants' pregnancies occurred soon after the WHO revised the diagnostic thresholds for GDM (2013). ${ }^{[26]}$ However, the National Department of Health's current policy on GDM still reflects the 1999 WHO diagnostic criteria. External factors, such as government policy and the training and experience of healthcare professionals, may affect the doctor-patient relationship. Doctors' views on the seriousness of GDM affect their patients' beliefs and health-related behaviour, as well as their understanding of the longterm consequences for the mother and her children. ${ }^{[22,27]}$ Uniformity in screening practices with access to screening at all healthcare centres may improve the safety of pregnancies.

Although it would have been more convenient to attend the antenatal service of the local community health clinic, participants were not confident that their GDM would have been identified there. In SA, diagnostic testing and treatment for GDM are available only at hospitals, which is not uncommon in low- and middleincome countries. ${ }^{[28,29]}$ In addition, GDM as defined by the 2013 diagnostic thresholds of the WHO is still not recognised as a distinct clinical entity in SA, and so treatment is not consistently offered in a non-research setting. Treatment of overt diabetes in pregnancy varies between hospitals. For example, at the CHBAH Diabetes in Pregnancy clinic, patients are managed by both endocrinologists and obstetricians at each visit. However, at the Rahima Moosa Mother and Child Academic Hospital in Johannesburg, which is also a teaching hospital associated with the University of the Witwatersrand, endocrinologists are not involved in the care of these patients. Patients are not always admitted for initiation of insulin therapy to treat GDM at second-level care centres in the region. GDM treatment is not available at community health clinics. ${ }^{[28]}$ Such inconsistency and variation in practice can only increase the potential for a negative reaction of patients to the condition and to the processes that surround it. Availability of GDM screening and feasible, sustainable treatment strategies applicable to low-resource settings are essential to reduce the morbidity associated with these high-risk pregnancies and to ensure continued interest and compliance from patients in their treatment.

\section{Gestational diabetes mellitus from the perspective of affected women}

At CHBAH, all newly diagnosed GDM patients are admitted as part of standard practice, not uncommon in low- and middle-income countries. ${ }^{[29]}$ This serves to rapidly restore euglycaemia by optimising medication doses, which includes the use of insulin. Participants were strongly against hospital admission and this aversion was exacerbated by abrasive attitudes of nursing staff, the shortage of beds and unpalatable hospital food. In addition to their awareness of the shortage of healthcare resources, the women were uncertain of the actual benefits of this experience. Admission for intermediate hyperglycaemia of GDM may not be essential, as up to $90 \%$ of those affected do not require medication. ${ }^{[30]}$ Given a rising disease prevalence and lower diagnostic thresholds, an increasing number of women will be diagnosed with GDM, which could lead to existing services being overwhelmed. The need for routine hospital admission should be re-evaluated when local guidelines for GDM diagnosis and management are reviewed.

Dietary advice is a core component of GDM interventions and diabetes prevention programmes. Participants described the prescribed diet as unpalatable, not aligned with their personal preferences and unsustainable in the long term. There are reports describing the GDM-prescribed diet as contrary to women's personal preferences, culturally insensitive and difficult to follow. ${ }^{[18,19,27,31-33]}$ The wellbeing of the unborn child and a history of pregnancy loss compelled women toward dietary adherence until the birth. However, clinicians' disregard for individual preferences and circumstances may not facilitate the sustained, lifelong change necessary to prevent GDM recurrence or the development of overt diabetes. A recent Cochrane review found no superiority between ten different diets in the primary outcome of the development of macrosomia in GDM-affected pregnancies, nor in the prevention of GDM development. ${ }^{[34,35]}$ There is no ideal, universally applicable diet for the treatment of GDM. Rather than adherence to a specific diet, avoiding excess maternal weight gain and lifestyle changes to restore euglycaemia are associated with a reduction in the perinatal morbidity of GDM..$^{[36-38]}$

Misunderstanding of the health information offered may be a reason why one of the participants believed that pap (maize meal) was not allowed at all in the GDM diet. GDM is a state of carbohydrate intolerance. Adjustments to the portion size and the quality of starch of the meal are important in curbing postprandial excursions in plasma glucose concentrations. Ideally, the starch portion of a meal should be restricted to $\sim 30 \mathrm{~g}$. This equates to a quarter of a standard $23 \mathrm{~cm}$ dinner plate, two slices of bread or a portion of pap the size of an individual's fist. ${ }^{[39]}$ Starch with a low glycaemic index (GI) and a higher fibre content is preferable. ${ }^{[39]}$ Unlike whole corn and coarsely ground corn, refined maize meal has a high GI, meaning that it leads to high postprandial plasma glucose levels. ${ }^{[40]}$ Adjusting the food preparation process (for example, cooking maize meal, letting it cool and then reheating it before eating) can create digestion-resistant starch ${ }^{[41]}$ thereby lowering the GI and consequently postprandial plasma glucose concentrations. Including resistant starch in the diet is also associated with a reduction in obesity, which is associated with the development of diabetes mellitus. ${ }^{[41]}$ Appropriate dietary counselling in an appropriate language and assessing the patient's understanding may reduce confusion and facilitate uptake of sustainable dietary changes by GDM-affected women.

Participants experienced anxiety, self-blame and guilt secondary to unresolved distress from previous pregnancy losses; similar observations have been described previously. ${ }^{[42,43]}$ Unresolved distress from their previous experiences affected their response to the GDM diagnosis and some were ambivalent about knowing their GDM status. Inconsistent healthcare messages from the research unit, doctors and nurses may have contributed to this. Pretest counselling prior to screening may reduce patients' anxiety. GDM awareness and skills training of doctors, midwives and lay health workers at all centres will allow for consistent healthcare messages.

\section{Fear of diabetes}

An unexpected finding was participants' perception that having diabetes is worse than living with HIV. This may have contributed 


\section{CASE REPORT}

to the ambivalence some participants expressed regarding knowing their GDM status. Type 2 diabetes mellitus was described as something hidden - a dangerous medical condition that was difficult to diagnose and treat, with potentially catastrophic consequences. Participants' impressions were that even if one was formally screened, the diagnosis may still be missed. The underlying cause of diabetes mellitus was poorly understood by participants, particularly by those who did not have a positive family history. In SA, HIV infection is the fifth leading cause of death, but the trend for this and other infectious diseases is decreasing. Diabetes is the leading cause of death of SA women and the second leading cause of all deaths, associated with an increasing trend for non-communicable diseases. Women may benefit from consistent healthcare information to reduce GDM-associated distress.

\section{Study limitations}

Participants directed the flow of discussions and subthemes were not consistently generated within each group. Findings that emerge from FGDs are not representative of the opinions or ideas of every individual member, nor can they be transferred to any population beyond the group. This is a known limitation of FGDs, as some participants may be more inclined to dominate the group. ${ }^{[4]]}$ The composition of the focus groups was restricted to a single-state sector sample from Soweto. Diverse social, economic and cultural differences were therefore unlikely. The combined experience of this group may adequately reflect that of an urban black SA population with poor obstetric history attending a tertiary health facility.

\section{Conclusion}

Women diagnosed with GDM have a high risk for recurrence in subsequent pregnancies and for the development of overt diabetes in either mother or child in later years. ${ }^{[45]}$ Women were aware of the association between developing diabetes and genetic predisposition, obesity and unhealthy lifestyle choices. There was little awareness of the development of diabetes being preventable. Perception of risk post GDM is known to affect lifestyle choices postpartum. ${ }^{[4]}$ There is evidence that sustained lifestyle changes aimed at healthy eating, weight loss and regular exercise prevent the onset of type 2 diabetes mellitus. ${ }^{[47,48]}$ An evidence-based WHO review of diet and exercise education programmes suggest community development campaigns (e.g. diabetes prevention) that target high-risk groups (e.g. pre-diabetic women) and include group-based activities for homogenous groups of individuals (e.g. urban black African women) to be effective, even in low-resource settings. ${ }^{[49]}$ Intercultural variations in perception of ideal weight gain and nutrition in pregnancy have been described. ${ }^{[50]}$ Failure to clarify and address these issues may sabotage the success of GDM interventions in a local population. Culturally tailored gestational weight gain interventions may be necessary to optimise health outcomes and the local acceptability of interventions.

\section{Declaration. None.}

Acknowledgements. The authors thank the participants from the S1000 cohort, the staff of Developmental Pathways to Health Reseach Unit and our research assistant, Ms Nandi Mtshali.

Author contributions. LMD was responsible for developing the methodology, conducting the investigation, analysing the data and writing the manuscript. EJB and SAN contributed to developing the methodology and data analysis, and reviewed earlier drafts of the manuscript.
Funding. The work reported here was supported by funding from the South African Medical Research Council (SAMRC) through its Division of Research Capacity Development under the SAMRC clinician researcher (MD $\mathrm{PhD}$ ) scholarship programme, funded by the South African National Treasury. The contents of this research article are the sole responsibility of the authors and do not necessarily represent the official views of the SAMRC or the funders. SAN is supported by the Department of Science and Technology and the National Research Foundation Centre of Excellence in Human Development at the University of the Witwatersrand, Johannesburg.

\section{Conflicts of interest. None.}

1. World Health Organization. Global Report on Diabetes 2016. Geneva: WHO, 2016.

2. International Association of Diabetes and Pregnancy Study Groups Consensus Panel. International Association of Diabetes and Pregnancy Study Groups recommendations on the diagnosis and classification of hyperglycemia in pregnancy. Diabetes Care 2010;33(3):676-682. https://doi org/10.2337/dc09-1848

3. Ratner RE. Prevention of type 2 diabetes in women with previous gestational diabetes. Diabetes Care 2007;30(Suppl 2):S242-S245. https://doi.org/10.2337/dc07-s223

4. Brown J, Alwan NA, West J, et al. Lifestyle interventions for the treatment of women with gestational diabetes. Cochrane Database Syst Rev 2017;2017(5):CD011970. https://doi.org/10.1002/14651858. CD011970.pub2

5. Kaptein S, Evans M, McTavish S, et al. The subjective impact of a diagnosis of gestational diabetes among ethnically diverse pregnant women: A qualitative study. Can J Diabetes 2015;39(2):117-122 https://doi.org/10.1016/j.jcjd.2014.09.005

6. Martis R, Crowther CA, Shepherd E, Alsweiler J, Downie MR, Brown J. Treatments for women with gestational diabetes mellitus : An overview of Cochrane systematic reviews. Cochrane Database Syst Rev 2018;8(8):CD012327. https://doi.org/10.1002/14651858.CD012327.pub2

7. Han S, Crowther CA, Middleton P. Interventions for pregnant women with hyperglycaemia not meeting gestational diabetes and type 2 diabetes diagnostic criteria. Cochrane Database Syst Rev 2012;1:CD009037. https://doi.org/10.1002/14651858.CD009037.pub2

8. Seidel M, Powell RO, Zgibor JC, Siminerio LM, Piatt GA. Translating the Diabetes Prevention program into an urban medically underserved community. Diabetes Care 2008;31(4):684-689. https://doi. org/10.2337/dc07-1869

9. Chapman E, Reveiz L, Sangalang S, et al. A survey study identified global research priorities for decreasing maternal mortality. J Clin Epidemiol 2014;67(3):314-324. https://doi.org/10.1016/j. jclinepi.2013.10.007

10. Attridge M, Creamer J, Ramsden M, Cannings-John R, Hawthorne K. Culturally appropriate health education for people in ethnic minority groups with type 2 diabetes mellitus. Cochrane Database Syst Rev 2014;9(9):CD006424. https://doi.org/10.1002/14651858.CD006424.pub3

11. World Health Organization. Interventions on diet and physical activity: What works. Summary report Geneva: WHO, 2009.

12. Kulkarni KD. Food, culture, and diabetes in the United States. Clin Diabetes 2007;22(4):190-192. https://doi.org/10.2337/diaclin.22.4.190

13. Pantoja T, Opiyo N, Lewin S, et al. Implementation strategies for health systems in low-income countries: An overview of systematic reviews. Cochrane Database Syst Rev 2017;(9):CD011084. https://doi.org/10.1002/14651858.CD011086.pub2

14. Moore G, Audrey S, Barker M, et al. Process evaluation of complex interventions: Medical Research Council guidance. BMJ 2015;350:h1258. https://doi.org/10.1136/bmj.h1258

15. May CR, Mair FS, Dowrick CF, Finch TL. Process evaluation for complex interventions in primary care: Understanding trials using the normalization process model. BMC Fam Pract 2007;8(1):42. https://doi.org/10.1186/1471-2296-8-42

16. Osborn CY, Amico KR, Cruz N, et al. Development and implementation of a culturally tailored diabetes intervention in primary care. Transl Behav Med 2011;1(3):468-479. https://doi.org/10.1007/ s13142-011-0064-9

17. Hennink MM, Kaiser BN, Sekar S, Griswold EP, Ali MK. How are qualitative methods used in diabetes research? A 30-year systematic review. Glob Public Health 2017;12(2):200-219. https://doi.org/10.10 80/17441692.2015.1120337

18. Lapolla A, Di Cianni G, Di Benedetto A, et al. Quality of life, wishes, and needs in women with gestational diabetes: Italian DAWN pregnancy study. Int J Endocrinol 2012;2012:784726. https://doi. org/10.1155/2012/784726

19. Morrison MK, Lowe JM, Collins CE. Australian women's experiences of living with gestational diabetes. Women Birth 2014;27:52-57. https://doi.org/10.1016/j.wombi.2013.10.001

20. Carolan-Olah MC. Educational and intervention programmes for gestational diabetes mellitus (GDM) management: An integrative review. Collegian 2016;23(1):103-114. https://doi.org/10.1016/j. colegn.2015.01.001

21. Gibson-Helm M, Teede H, Block A, et al. Maternal health and pregnancy outcomes among women of refugee background from African countries: A retrospective, observational study in Australia. BMC Pregnancy Childbirth 2014;14:392. https://doi.org/10.1186/s12884-014-0392-0

22. Hjelm K, Bard K, Apelqvist J. A qualitative study of developing beliefs about health, illness and healthcare in migrant African women with gestational diabetes living in Sweden. BMC Womens Health 2018;18(1):34. https://doi.org/10.1186/s12905-018-0518-z

23. Dickson LM, Buchmann EJ, Janse van Rensburg C, Norris SA. Accuracy of five plasma calibrated glucometers to screen for and diagnose gestational diabetes mellitus in a low resource clinic setting. J Clin Transl Endocrinol 2019;16:100174. https://doi.org/10.1016/j.jcte.2018.12.003

24. Dickson LM, Buchmann EJ, Janse van Rensburg C, Norris SA. The impact of differences in plasm glucose between glucose oxidase and hexokinase methods on estimated gestational diabetes mellitus prevalence. Sci Rep 2019;9(1):7238. https://doi.org/10.1038/s41598-019-43665-x

25. Dorland K, Liddy C. A pragmatic comparison of two diabetes education programs in improving type 2 diabetes mellitus outcomes. BMC Res Notes 2014;7:186. https://doi.org/10.1186/1756-0500-7-186

26. World Health Organization. Diagnostic Criteria and Classification of Hyperglycaemia First Detected in Pregnancy. Geneva, WHO, 2013.

27. Draffin CR, Alderdice FA, McCance DR, et al. Exploring the needs, concerns and knowledge of women diagnosed with gestational diabetes: A qualitative study. Midwifery 2016;40:141-147. https:// doi.org/10.1016/j.midw.2016.06.019 


\section{CASE REPORT}

28. South African National Department of Health. Guidelines for Maternity Care in South Africa A manual for clinics, community health centres and district hospitals. Pretoria: DoH, 2015. http://www.health-e.org.za/wp-content/uploads/2015/11/Maternal-Care-Guidelines-2015_FINAL21.7.15.pdf

29. Utz B, Kolsteren P, De Brouwere V. A snapshot of current gestational diabetes management practices from 26 low-income and lower-middle-income countries. Int J Gynecol Obstet 2016;134(2):145 150. https://doi.org/10.1016/j.ijgo.2016.01.020

30. Brown J, Alwan N, West J, et al. Lifestyle interventions for treatment of women with gestational diabetes (Review). Cochrane Database Syst Rev 2017;(5):CD011970. https://do org/10.1002/14651858.CD011970.pub2

31. Hui AL, Sevenhuysen G, Harvey D, Salamon E. Food choice decision-making by women with gestational diabetes. Can J Diabetes 2014;38:26-31. https://doi.org/10.1016/j.jcjd.2013.08.26

32. Carolan M, Steele C, Margetts H. Knowledge of gestational diabetes among a multi-ethnic cohort in Australia. Midwifery 2010;26(6):579-588. https://doi.org/10.1016/i.midw.2009.01.006

33. Hui AL, Sevenhuysen G, Harvey D, Salamon E. Barriers and coping strategies of women with gestational diabetes to follow dietary advice. Women Birth 2014;27(4):292-297. https://doi. org/10.1016/j.wombi.2014.07.00

34. Han S, Middleton P, Shepherd E, Van Ryswyk E, Crowther CA. Different types of dietary advice for women with gestational diabetes mellitus. Cochrane Database Syst Rev 2017;2(2):CD009275. https://doi.org/10.1002/14651858.CD009275.pub3

35. Tieu J, Shepherd E, Middleton P, Crowther CA. Dietary advice in pregnancy for preventing gestational diabetes mellitus. Cochrane Database of Systematic Rev 2017;(1):CD006674

36. Crowther CA, Hiller JE, Moss JR, et al. Effect of treatment of gestational diabetes mellitus on pregnancy outcomes. N Engl J Med 2005;352:2477-2486. https://doi.org/10.1056/NEJMoa042973

37. Muktabhant B, Lawrie TA, Lumbiganon P, Laopaiboon M. Diet or exercise, or both, for preventing excessive weight gain in pregnancy. Cochrane Database Syst Rev 2015;6(6):CD007145. https://do org/10.1002/14651858.CD007145.pub3

38. Kaiser B, Jeannot E, Razurel C. Determinants of health behaviors after gestational diabetes mellitus: A prospective cohort study in Geneva. J Midwifery Womens Health 2016;61(5):571-577. https:/ doi.org/10.1111/jmwh. 12486

39. Centers for Disease Control and Prevention. Diabetes meal planning. 2020. https://www.cdc.gov/ diabetes/managing/eat-well/meal-plan-method.html. (accessed 14 April 2020)

40. Mlotha V, Mwangwela AM, Kasapila W, Siyame EW, Masamba K. Glycemic responses to maize flou stiff porridges prepared using local recipes in Malawi. Food Sci Nutr 2015;4(2):322-328. https://doi. stiff porridges prepared
org $/ 10.1002 \% 2 \mathrm{Ffsn} 3.293$
41. Birt DF, Boylston T, Hendrich S, et. al. Resistant starch: Promise for improving human health. Adv Nutr 2013;4(6):587-601. https://doi.org/10.3945/an.113.004325

42. Woods-Giscombé CL, Lobel M, Crandell JL. The impact of miscarriage and parity on patterns of maternal distress in pregnancy, Res Nurs Health 2010;33(4):316-328. https://doi. org/10.1002\%2Fnur.20389

43. Gravensteen IK, Jacobsen EM, Sandset PM, et al. Anxiety, depression and relationship satisfaction in the pregnancy following stillbirth and after the birth of a live-born baby: A prospective study. BMC Pregnancy Childbirth 2018;18(1):41. https://doi.org/10.1186\%2Fs12884-018-1666-8

44. Onwuegbuzie AJ, Dickinson WB, Leech NL, Zoran AG. A qualitative framework for collecting and analyzing data in focus group research. Int J Qual Methods 2009;8(3):1-21.

45. Hod M, Kapur A, Sacks DA, et al. The International Federation of Gynecology and Obstetrics (FIGO) Initiative on gestational diabetes mellitus: A pragmatic guide for diagnosis, management, and care. Int J Gynecol Obstet 2015;131:S173-211. https://doi.org/10.1016 S0020-7292(15)30033-3

46. Kim C, McEwen LN, Piette JD, Goewey J, Ferrara A, Walker EA. Risk perception for diabete among women with histories of gestational diabetes mellitus. Diabetes Care 2007;30(9):2281 2286. https://doi.org/10.1016/j.bpobgyn.2014.04.019

47. Chasan-Taber L. Lifestyle interventions to reduce risk of diabetes among women with prio gestational diabetes mellitus. Best Pract Res Clin Obstet Gynaecol 2015;29(1):110-122. https:// doi.org/10.1016/j.bpobgyn.2014.04.019

48. Bentley-Lewis R, Levkoff S, Stuebe A, Seely EW. Gestational diabetes mellitus: Postpartum opportunities for the diagnosis and prevention of type 2 diabetes mellitus. Nat Clin Pract Endocrinol Metab 2008;4(10):552-558. https://doi.org/10.1038/ncpendmet0965

49. World Health Organization. Diet, Nutrition and the Prevention of Chronic Diseases. Geneva: WHO, 2003.

50. Whitaker KM, Wilcox S, Liu J, Blair SN, Pate RR. African American and White women's perceptions of weight gain, physical activity, and nutrition during pregnancy. Midwifery 2016;34:211-220. https://doi.org/10.1016/j.midw.2015.11.005

Accepted 25 April 2020 